\title{
Beitragsserien
}

\section{Raumbezug in der Umweltbeobachtung des Bundes und der Länder}

\author{
Vorwort* \\ Gerlinde Knetsch
}

Korrespondenzadresse: Gerlinde Knetsch, Umweltbundesamt, Fachgebiet Umweltbeobachtung, Postfach 330022 , D-14191 Berlin; e-mail: gerlinde.knetsch@uba.de

\section{Zielsetzung}

Ausgehend von der Tatsache, dass die von Bund und Ländern betriebenen Messnetze größtenteils auf einzelne Umweltmedien ausgerichtet sind, besteht das Ziel der Umweltbeobachtung darin, eine Verknüpfung dieser Messnetze je nach fachlichem Erfordernis und umweltpolitischem Bedarf unter dem Blickwinkel einer medienübergreifenden Betrachtung zu erreichen. Unverzichtbare Werkzeuge dazu sind statistische Verfahren und Modelle, die es ermöglichen, Daten aus der Umweltbeobachtung auf ihre Repräsentativität hin zu untersuchen und - wenn möglich - räumlich und zeitlich zu verallgemeinern.

Eine Methode, dieses Ziel zu erreichen, besteht in der Anwendung eines Raumgliederungsverfahrens. Ziel einer Raumgliederung für Umweltbeobachtung ist es, Naturräume auszuweisen, die bei ausgewählten Standortmerkmalen (Klima, Boden, Orographie, Vegetation) primär zwei Kriterien erfüllen:

- Homogenität, das heißt, die Standortmerkmale sollen innerhalb eines Naturraumes möglichst homogen ausgeprägt sein und

- Trennschärfe, das heißt, die Naturräume sollen sich bezüglich der Merkmalsausprägungen weitgehend unterscheiden.

\section{Vorgehensweise}

Unter Anwendung von multivariat-statistischer Verfahren und deren Einbindung in ein Geographisches Informationssystem (GIS) können Gebiete in verschiedene Naturräume (Standorttypen) unterteilt werden. Hierarchische Verfahren ermöglichen es, verschiedene Ebenen einer solchen Regionalisierung abzubilden. Das bedeutet, dass die Raumeinheiten einer höheren Gliederungsebene nach Maßgabe der Kriterien Homogenität und Trennschärfe auf tieferen Ebenen weiter unterteilt werden.

Jeder der so definierten Raumeinheiten repräsentiert einen entsprechenden Naturraumtyp, der durch ähnlich ausgeprägte Standortmerkmale charakterisiert ist, wobei die Ähnlichkeit (Homogenität) mit der Gliederungstiefe zunimmt. Durch Überlagerung einer solchen Raumgliederung mit Messnetzinformationen der verschiedenen Umweltbeobachtungsprogramme (z.B. geografische Lage, Messgrößen und -methoden) lassen sich für diese Naturräume erste Aussagen hinsichtlich der Dichte, des Beobachtungsspektrums und der Methodik von Mess- und Beobachtungsnetzen treffen. Ferner können Erkenntnisse zu inhaltlichen und räumlichen Redundanzen oder Lücken und zum möglichen Harmonisierungsbedarf der Messnetze abgeleitet werden. Mit dem Einsatz einer Raumgliederung kann somit ein erster Schritt volizogen werden, die bisher überwiegend sektoral ausgerichteten Umweltbeobachtungsprogramme in Beziehung zu setzen, ihre Repräsentativität zu untersuchen und bei Vorliegen von geeigneten Kriterien mit einander zu verknüpfen. In einem weiteren Schritt können die an den einzelnen Messnetzstandorten erhobenen Daten mit geostatistischen Verfahren auf ihre räumliche Repräsentanz überprüft werden.

\section{Anwendungsgebiete}

Im intensiven fachlichen Austausch mit Behörden und Instituten, an dem unter anderem das Bundesministerium für Umwelt, $\mathrm{Na}$ turschutz und Reaktorsicherheit, das Bundesamt für Naturschutz, das Statistische Bundesamt, die Bundesanstalt für Geowissenschaften und Rohstoffe sowie das Umweltbundesamt teilnahmen, findet das Thema Raumbezug und Umweltbeobachtung Eingang in vier Arbeiten. Sie befassen sich einerseits mit der Regionalisierung Deutschlands, andererseits stehen räumlich begrenze Gebiete wie z.B. Biosphärenreservate - im Interesse der Untersuchungen.

\section{Literatur}

BMU - Bundesministerium für Umwelt, Naturschutz und Reaktorsicherheit (1999): Konzept Ökologische Unweltbeobachtung des Bundes und der Länder. Bonn

SCHRoder, W.; SCHMidt, G.; Ahrens, E.; BarTels, F.; SCHMidT, B. (1999): Entwicklung eines Modells zur Zusammenführung vorhandener Daten des Bundes und der Länder zu einem Umweltbeobachtungssystem. Bd. 1: Instrumentarium für die Zusammenführung umweltrelevanter Daten verschiedener Messnetze. Bd. 2: Konzept und Realisierung der Standörtlichen Gliederung Deutschlands für die Ökologische Umweltbeobachtung. Kiel, Vechta (Abschlußbericht FuEVorhaben 29781 126/01, im Auftrag des Umweltbundesamtes)

Die Ergebnisse dieser Arbeiten werden in einer Beitragsserie vorgestellt:

Vorwort: Gerlinde Knetsch

Teil I: Schröder, W.; Schmidt, G.: Raumgliederung für die Ökologische Umweltbeobachtung des Bundes und der Länder (Hochschule Vechta, Institut für Umweltwissenschaften)

Teil П: Schäfer, D.; Seibel, S.; Hoffmann-Kroll, R.: Raumbezug und Repräsentativität der Ökologischen Flächenstichprobe (Statistisches Bundesamt)

Teil III: Vahrson, W.-G.; Dreger, F.; Luthardt, V.: Flächenauswahl und ökosystemares Monitoring in den Biosphärenreservaten Schorfheide-Chorin und Spreewald (Fachhochschule Eberswalde, Fachbereich Landschaftsnutzung und Naturschutz)

Teil IV: Schönthaler, K.: Raumbezug in der ökosystemaren Umweltbeobachtung - Anforderungen an die modellhafte Umsetzung im Biosphärenreservat Rhön (Bosch \& Partner GmbH)

* Ein ausführliches Vorwort finden Sie unter DOI: http://.dx.doi.org/1065.65/uwst2000.05.019 